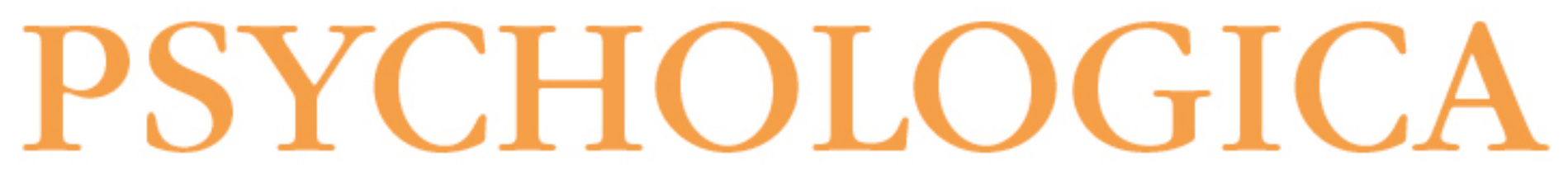

Adaptação e validação da Eustress Scale para professores portugueses

Autor(es): $\quad$ Fonseca, Susana; Jordão, Filomena

Publicado por: Imprensa da Universidade de Coimbra

URL

persistente: URI:http://hdl.handle.net/10316.2/35824

DOI: $\quad$ DOI:http://dx.doi.org/10.14195/1647-8606_57_1_6

Accessed : $\quad$ 26-Apr-2023 12:13:22

A navegação consulta e descarregamento dos títulos inseridos nas Bibliotecas Digitais UC Digitalis, UC Pombalina e UC Impactum, pressupõem a aceitação plena e sem reservas dos Termos e Condições de Uso destas Bibliotecas Digitais, disponíveis em https://digitalis.uc.pt/pt-pt/termos.

Conforme exposto nos referidos Termos e Condições de Uso, o descarregamento de títulos de acesso restrito requer uma licença válida de autorização devendo o utilizador aceder ao(s) documento(s) a partir de um endereço de IP da instituição detentora da supramencionada licença.

Ao utilizador é apenas permitido o descarregamento para uso pessoal, pelo que o emprego do(s) título(s) descarregado(s) para outro fim, designadamente comercial, carece de autorização do respetivo autor ou editor da obra.

Na medida em que todas as obras da UC Digitalis se encontram protegidas pelo Código do Direito de Autor e Direitos Conexos e demais legislação aplicável, toda a cópia, parcial ou total, deste documento, nos casos em que é legalmente admitida, deverá conter ou fazer-se acompanhar por este aviso. 
VOLUME $5 \longdiv { 2 0 1 4 }$

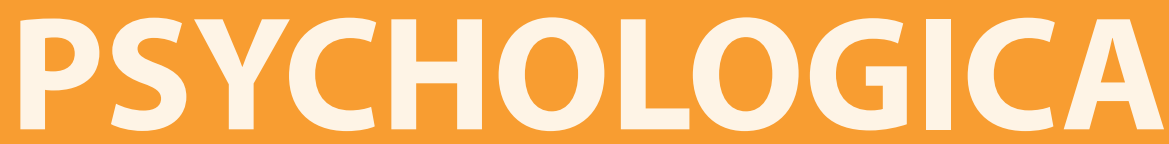

IMPRENSA DA UNIVERSIDADE DE COIMBRA

COIMBRA UNIVERSITY PRESS

FACULDADE DE PSICOLOGIA E DE CIÊNCIAS

DA EDUCAÇÃO DA UNIVERSIDADE DE COIMBRA 


\title{
Adaptação e validação da Eustress Scale para professores portugueses
}

\author{
Susana Fonseca ${ }^{1}$ e Filomena Jordão ${ }^{2}$
}

Adaptation and validation study of the Eustress Scale for Portuguese teachers

\begin{abstract}
Teachers' stress has been widely studied, but the focus has been predominantly in dysfunctional aspects. Several studies point out that stress can be a positive psychological response to a stressor, referred as eustress (Campbell-Quick, Cooper, Nelson, Quick, \& Gavin, 2003; Simmons \& Nelson, 2001; Simmons, Nelson, \& Quick, 2003).

A sample of 496 Portuguese teachers was evaluated and the main aim of the study was to adapt and validate the eustress scale developed by O'Sullivan (2011) for Portuguese teachers.

The results show that this scale has adequate psychometric properties with a high value of internal consistency $(\alpha=.93)$, similar to the results of a previous independent sample (Fonseca \& Jordão, 2011). The factor that resulted from the exploratory factor analysis explains $78,87 \%$ of the total variance.

More studies should be conducted to validate the eustress scale, in teachers and also the general Portuguese population, to examine, in particular, the stability of this scale.
\end{abstract}

Keywords: eustress scale; psychometric analysis; Portuguese teachers; occupational stress

1 Susana Fonseca, Faculdade de Psicologia e de Ciências da Educação, Universidade do Porto. E-mail: susanafonseca@ esev.ipv.pt

2 Filomena Jordão, Faculdade de Psicologia e de Ciências da Educação, Universidade do Porto. 


\section{Resumo}

O stress dos professores tem sido largamente estudado, mas, o seu enfoque tem sido predominantemente direcionado para a sua vertente disfuncional. Contudo, diversos estudos indicam que o stress pode apresentar-se como uma resposta psicológica positiva a um stressor, designando-se de eustress (Campbell-Quick, Cooper, Nelson, \& Gavin, 2003; Simmons \& Nelson, 2001; Simmons, Nelson, \& Quick, 2003).

Este estudo foi realizado numa amostra de 496 professores e teve como principal objetivo adaptar e validar a eustress scale, desenvolvida por O'Sullivan (2011), para professores portugueses.

Os resultados evidenciam que a escala possui qualidades psicométricas adequadas, com um valor elevado de consistência interna $(\alpha=.93)$, semelhante ao encontrado anteriormente com uma amostra independente (Fonseca \& Jordão, 2011). O fator resultante da análise fatorial exploratória explica $78,87 \%$ da variância total.

Mais estudos devem ser realizados para validar a escala de eustress, nos professores e para a população portuguesa em geral, analisando, nomeadamente, a estabilidade da escala.

Palavras-chave: escala de eustress; análise psicométrica; professores portugueses; stress ocupacional

\section{INTRODUÇÃO}

O stress ocupacional tem sido visto como um problema decorrente do mundo industrializado (McGowan, Gardner \& Fletcher, 2006), cuja prevalência tem aumentado significativamente nas últimas décadas e as suas consequências negativas na saúde e no bem-estar de pessoas e organizações também. Os estudos nesta área têm proliferado nos últimos vinte anos, e uma base sólida de conhecimento tem sido construída, centrada na identificação dos stressores, das respostas individuais e das consequências do distress (Nelson \& Simmons, 2005).

O stress dos professores, decorrente das dificuldades sentidas pelos mesmos em lidar com as diversas alterações que têm tido lugar no contexto de ensino, constitui de há alguns anos para cá uma fonte de preocupação não só para os docentes, mas também para a comunidade educativa em geral, os investigadores, os responsáveis políticos e para a sociedade em geral (Mota-Cardoso, Araújo, Ramos, Gonçalves, \& Ramos, 2002; Travers \& Cooper, 1996).

Diversos estudos têm procurado estudar o fenómeno do stress nos professores, focando-se na vertente tradicional da prevenção dos aspetos nefastos do stress. 
Contudo, esta atenção dada aos aspetos negativos do stress orienta-se apenas para uma parte do fenómeno, na medida em que o stress pode produzir simultaneamente respostas e resultados positivos (Nelson \& Simmons, 2003). Esta perspetiva remonta à conceptualização original de stress apresentada por Selye (1976) e acrescenta as respostas afetivas positivas ao processo de stress, designando-as de "eustress".

$\mathrm{O}$ eustress reflete até que ponto uma situação ou evento é avaliado cognitivamente como podendo beneficiar o indivíduo ou melhorar o seu bem-estar (resposta positiva), sendo expectável que a maioria das situações de trabalho evoque um conjunto misto de respostas positivas e negativas (Simmons \& Nelson, 2007). Contudo, estas respostas são independentes, distintas, multivariadas e na sua essência potencialmente interativas (Simmons \& Nelson, 2007).

Assim, não é compreensível que se assuma a presença de eustress através da simples observação de ausência de distress. Consequentemente, a avaliação independente do eustress torna-se fundamental para uma melhor compreensão do conceito, o que permitirá compreender as estratégias que potenciam vivências de estados psicológicos positivos decorrentes de situações e eventos stressantes, promovendo assim, o bem-estar do trabalhador e da organização.

É neste sentido, conscientes da inexistência de instrumentos de avaliação do eustress traduzidos e adaptados para a população portuguesa, em geral, e para os professores, especificamente, que nos propomos a adaptar e a avaliar as propriedades psicométricas de um instrumento de avaliação do eustress e a descrever a forma como o eustress se manifesta na amostra de professores em estudo.

\section{STRESS OCUPACIONAL}

De acordo com o modelo transaccional de stress desenvolvido por Lazarus e Folkman (1984), o stress ocupacional consiste na relação que se estabelece entre o ambiente de trabalho e as características do indivíduo que, em determinada altura, e por um processo de avaliações cognitivas da situação e dos recursos disponíveis, verifica que as exigências do trabalho excedem a sua capacidade em lidar com elas (Cooper, Dewe, \& Driscoll, 2001). Este modelo viria mais tarde a ser reformulado, no sentido de dar conta da possibilidade de, numa situação de stress, poderem ocorrer simultaneamente emoções positivas e negativas (Folkman, 2008).

O stress ocupacional tem sido amplamente estudado na Psicologia das Organizações, mas o seu enfoque tem sido predominantemente direcionado para a sua vertente disfuncional. Mais recentemente, motivados pelo movimento da Psicologia Positiva, impulsionado por Seligman e Csikszentmihalyi (2000), Nelson e Simmons (2003) 
propõem um novo modelo de stress ocupacional, o Modelo Holístico de Stress, teoricamente ancorado na perspetiva transacional de stress (Lazarus \& Folkman, 1984). Nelson e Simmons (2003) apresentam, assim, o seu modelo como um modelo complexo, onde o eustress é conceptualizado como algo mais do que a mera ausência de distress, sugerindo que a presença ou ausência de cada um deles é necessária para avaliar completamente a resposta de stress.

O Modelo Holístico de Stress (Nelson \& Simmons, 2003) vem assim completar a compreensão sobre a experiência individual de stress ocupacional, uma vez que engloba quer as respostas positivas (eustress), quer as respostas negativas (distress) às exigências do trabalho. De acordo com este modelo, a vertente positiva ou negativa dos stressores depende da avaliação cognitiva que o indivíduo faz dos mesmos. Estas formas de avaliação concorrem para identificar até que ponto cada acontecimento é avaliado como perigoso/ameaçador ou desafiante. Desta forma, os fatores promotores de stress raramente são percebidos como puramente positivos ou negativos. Nesta perspetiva o eustress reflete o grau em que o indivíduo percebe, com base na avaliação cognitiva que faz, uma situação especialmente exigente como benéfica ou promotora do seu bem-estar.

Contudo, poucos estudos podem ser encontrados na literatura sobre o constructo eustress. Este facto pode ser atribuído, por um lado, à ampla e predominante atenção que tem recebido a vertente negativa do stress (Edwards \& Cooper, 1988) e, por outro, à constante mudança na definição, operacionalização e medição do referido constructo (Little, Simmons e Nelson, 2007).

\section{Evolução e definição do conceito de eustress}

Hans Selye (1974) foi o primeiro autor a apresentar o termo eustress, incorporando-o como um elemento da sua teoria sobre o stress humano, denotando que nem todos os estados de stress são nocivos, havendo dois tipos de stress que diferem no seu impacto junto do indivíduo. O distress que é prejudicial e nocivo, relaciona-se com a diminuição das capacidades do indivíduo, determinando uma sensação de impotência face às dificuldades e consequente má adaptação à situação de exigência vivenciada (Selye, 1987). O eustress é benéfico e construtivo, estando associado a uma quantidade de stress que nos mantém interessados pela vida e nos faz enfrentar desafios, face às exigências. Deste modo, o indivíduo que vivencia eustress estará mais autoconfiante e terá maior probabilidade de ultrapassar as dificuldades (Selye, 1987. O autor considerava que estados breves, suaves e controlados de desafio à homeostasia, poderiam ser vivenciados como agradáveis ou estimulantes, podendo incentivar o desenvolvimento e o crescimento intelectual e emocional. 
Quick e colaboradores (1997), sob a influência dos trabalhos desenvolvidos por Selye, definiram a resposta de stress como uma resposta generalizada, padronizada, uma mobilização inconsciente dos recursos energéticos naturais do corpo quando confrontado com uma exigência ou stressor. Os autores definem eustress como o resultado construtivo, positivo e saudável de um evento stressante e como uma resposta positiva ao stress (Quick et al., 1997). Contudo, o trabalho destes autores acerca do eustress representa poucos avanços teóricos e empíricos em relação aos já apresentados por Selye. E apesar de o modelo, por eles apresentado, incorporar o conceito de eustress como um efeito separado e distinto, encaram-no essencialmente como a ausência de distress. A perspetiva dos autores sobre o stress baseia-se, assim, na Lei de Yerkes-Dodson, que patenteia que o aumento do stress é benéfico para a performance quando níveis ótimos são alcançados, a partir dos quais a performance decai (Benson \& Allen, 1980; Certo, 2003; Le Fevre, Matheny, $\&$ Kolt, 2003). Assim, nesta perspetiva, as pessoas que experienciam um certo nível de stress podem ser mais produtivas do que se o seu stress for eliminado.

A par do modelo apresentado por Quick e colaboradores (1997) vários outros modelos têm entretanto surgido na literatura sobre o stress ocupacional (Cooper, 1998), dos quais destacamos, devido à sua prevalência e centralidade, o Modelo de Ajustamento Pessoa-Ambiente (Edwards, Caplan, \& Van Harrison, 1998), o Modelo Cibernético (Cummings \& Cooper, 1998) e o Modelo Exigências do Trabalho-Controlo (Karasek, 1979). Contudo, o seu contributo para a definição, quer conceptual quer operacional, do eustress foi praticamente nulo.

Assim, no que diz respeito ao contributo destes modelos para o desenvolvimento do conceito de eustress podemos referir que, o Modelo de Ajustamento Pessoa-Ambiente assume implicitamente que o stress é distress e não menciona sequer o conceito de eustress. A abordagem cibernética refere-se ao stress mas não especifica se é distress ou eustress, contudo, ao salientar a capacidade de o individuo interpretar os stressores permite-nos inferir a existência de eustress, mas a teoria por si só não fornece ligações explícitas ao conceito. O Modelo de Controlo salienta que a perceção de controlo da pessoa sobre o stressor é uma variável importante, na medida em que modera a experiência de stress. Mais uma vez, a partir deste modelo, apenas podemos inferir a existência de eustress.

No sentido de estudar o eutress, Le Fevre e colaboradores (2003) apresentam uma revisão do modelo de controlo de Karasek (1979), onde salientam que o eustress ou o distress experienciado pelo individuo decorrente da perceção que tem acerca do stressor, deriva em consequências comportamentais, físicas e /ou psicológicas para o mesmo.

Tem havido, por parte de diversos académicos, alguma preocupação na definição conceptual do eustress, contudo essa preocupação é quase inexistente no que concerne à sua definição operacional. O eustress (do grego eu, significa bom), é 
operacionalizado, de acordo com o modelo holístico de stress (Simmons, 2000; Nelson \& Simmons, 2003), como uma resposta psicológica positiva a um stressor, como indicador da presença de estados psicológicos positivos, refletindo em que medida a avaliação cognitiva de uma situação é vista como benéfica para o indivíduo ou como potenciando o seu bem-estar.

Este conceito tem sido definido quer como um processo (Selye, 1976; Le Fevre, Matheny, \& Kolt, 2003) que implica responder positivamente ao stress, quer como uma consequência desse processo (Quick, Quick, Nelson, \& Hured, 1997), quer ainda como um processo e uma consequência/produto (O'Sullivan, 2011; Nelson \& Simmons, 2003, 2005; Simmons, 2000; Simmons \& Nelson, 2001). Este constructo é considerado como distinto do constructo de distress, definido como uma resposta psicológica negativa a um stressor, como indicador da presença de estados psicológicos negativos (Simmons, 2000; Nelson \& Simmons, 2003).

\section{Avaliação do eustress}

Se a definição conceptual de eustress carece ainda de aperfeiçoamento, a sua definição operacional tem sido largamente ignorada, sendo escassos os estudos que o procuram medir e os que o fazem, recorrem a diversos indicadores para o inferirem (Simmons, 2000; Simmons \& Nelson, 2001; Nelson \& Simmons, 2003, 2005).

Edwards e Cooper (1988) têm procurado estudar o eustress associando-o a estados psicológicos positivos e sugerindo a utilização desses estados para medir o constructo. Neste sentido, Simmons e Nelson (2001) utilizam a esperança, o meaningfulness, e o afeto positivo como estados psicológicos positivos para representar o eustress. Em 2004 os autores acrescentam o estado psicológico positivo manageability, como indicador de eustress, à sua investigação. Little, Simmons e Nelson (2007) acrescentam ainda, aos referidos indicadores, o comportamento de perdão.

Apenas em 2011 Geraldine O’Sullivan se propôs a criar uma escala para medir este constructo que define quer como o processo de responder positivamente ao stress, quer como a consequência positiva desse processo (O’Sullivan, 2011). Deste modo, a adaptação e avaliação das características psicométricas da Eustress Scale (O’Sullivan, 2011), revela-se fundamental, para uma melhor compreensão deste conceito.

\section{A Eustress Scale}

A escala de eustress desenvolvida por Geraldine O'Sullivan (2011) constitui-se como uma ferramenta válida para medir o eustress. Criada tendo por base a Perceived Stress Scale, desenvolvida por Cohen e colaboradores (1983), a escala de eustress é 
constituída por 15 itens, cinco dos quais são questões de despiste. Os itens incluem questões tais como: How often do you effectively cope with stressful changes that occur in your academic life? ${ }^{3}$ Aos participantes no estudo foi pedido que respondessem às questões considerando uma escala tipo Likert de seis pontos: "Never", "Almost Never", "Sometimes", "Often", "Very often" e "Always", em que os scores mais elevados indicam níveis de eustress mais elevados. O instrumento foi administrado a estudantes, em dois momentos distintos e para testar a fiabilidade da medida de eustress a autora realizou análises estatísticas cujos resultados indicaram coeficientes alpha de Cronbach de .766 e .806, na primeira e segunda administração respetivamente. Estes resultados refletem uma razoável consistência interna da escala alcançada sem ter sido necessário retirar nenhum item da escala. Foi ainda realizado um teste t para amostras emparelhadas, para analisar a consistência ao longo do tempo, que revelou a não existência de diferenças significativas entre a primeira e a segunda administração do instrumento $(\mathrm{t})=-.418, p=.679)$ (O’Sullivan, 2011).

\section{O STRESS NOS PROFESSORES}

Grande parte dos estudos realizados até ao momento, sobre o stress dos professores, tem considerado apenas as respostas negativas ao stress e os seus efeitos nefastos (insatisfação, doença, burnout, etc.). Desta forma, o stress ocupacional na profissão docente é descrito por Kyriacou e Sutcliffe (1979) como um síndroma de afetos negativos (como a raiva ou a depressão), associados recorrentemente a mudanças fisiológicas potencialmente patogénicas (p. ex., aumento do ritmo cardíaco), como resposta a aspetos do trabalho do professor e mediado pela perceção de que as exigências com que se confronta constituem ameaças ao seu bem-estar e à sua autoestima e mediado também por mecanismos de coping tendentes a reduzir a ameaça percecionada.

Em termos gerais, a investigação realizada no âmbito do stress nos professores associa diversas fontes de stress ocupacional (p. ex., ensinar alunos com falta de motivação, exigências de tempo, lidar com mudanças, ser avaliado por outros, fracas condições de trabalho) a manifestações (comportamentais, psicológicas e fisiológicas) resultantes do mesmo (Gomes et al., 2006; Kyriacou, 1987; Kyriacou, 1998; Kyriacou, 2001; Maia, 2004; Mota-Cardoso et al., 2002; Travers \& Cooper, 1996).

3 Posteriormente traduzido para: "Com que frequência supera eficazmente mudanças stressantes que ocorrem na sua vida académica?"

4 Posteriormente traduzido para "Nunca", “Quase nunca”, "Às vezes", "Frequentemente", "Muito Frequentemente” e "Sempre”. 
Os professores são confrontados no seu dia a dia com um crescente e constante conjunto de exigências e estão sob os olhares críticos da sociedade, facto que contribui para que a docência seja associada a níveis de stress elevados (Gomes \& Cruz, 2004; Melo, Gomes, \& Cruz, 1997; Mota-Cardoso et al., 2002). E, tal como reconhece Teodoro (1994), em Portugal a profissão docente constitui uma atividade que está sujeita a uma grande instabilidade, sobretudo para os seus membros mais jovens, e a um desgaste físico e psíquico permanente, particularmente percetível após os 15 a 20 anos de serviço docente.

As alterações com que se deparam os professores têm, assim, contribuído para o aumento e propagação do stress na profissão docente, como demonstram e salientam os estudos realizados em diferentes países (Gomes et al., 2006; Kyriacou, 1987; Kyriacou, 1998; Kyriacou, 2001; Maia, 2004; Mota-Cardoso et al., 2002; Travers \& Cooper, 1996; Venâncio, Carmo, Mendes, Liberato, \& César, 2000).

Contudo, como referimos anteriormente, o stress também pode ser benéfico, como comprovam estudos realizados com outros profissionais (p. ex., enfermeiros). Estes estudos têm salientado a existência de respostas positivas ao stress e dos seus efeitos benéficos para o indivíduo (satisfação no trabalho, bem-estar subjetivo, saúde, etc.) (Bishayee, 2012; Gillespie, Walsh, Winefield, Dua, \& Stough, 2001; Nelson \& Simmons, 2003; Simmons, 2000; Simmons \& Nelson, 2007; Simmons, Nelson, \& Quick, 2003). Deste modo, revela-se fundamental validar a escala de eustress para os professores portugueses e avaliar as suas características psicométricas.

\section{ESTUDO EMPÍRICO}

\section{Objetivos}

O objetivo principal desta investigação centra-se na adaptação, validação e exploração das propriedades psicométricas da Eustress Scale de O’ Sullivan, aplicada a professores portugueses. Procurar-se-á, deste modo, salvaguardando os requisitos científicos do processo de tradução, verificar a consistência interna e explorar a estrutura fatorial da escala quando aplicada a professores portugueses.

É ainda nosso objetivo descrever o nível de eustress percecionado pelos professores inquiridos, perceber se existem diferenças em função das variáveis pessoais e profissionais consideradas e conhecer a relação entre as variáveis em estudo. 


\section{Método}

\section{Amostra}

A amostra do nosso estudo foi recolhida por conveniência e é constituída por 496 professores (410 do sexo feminino, correspondendo a $82,7 \%$ da amostra e 86 do sexo masculino, isto é, $17,3 \%$ da amostra $)^{5}$ de diversas instituições (93,8\% instituições públicas e $6,3 \%$ instituições privadas $)^{6}$ e níveis de ensino (27\% do $1^{\circ} \mathrm{CEB}, 23 \%$ do $2^{\circ} \mathrm{CEB}, 45 \%$ do $3^{\circ} \mathrm{CEB}$ e Secundário, $1 \%$ do Ensino Superior Politécnico e 4\% do Ensino Superior

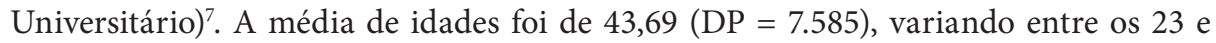
os 64 anos. Relativamente ao número de anos de serviço a média foi de 19,57 (DP = 8.68), com um número mínimo de anos de serviço de 1 e um máximo de 38 . O número médio de anos de serviço no atual nível de ensino foi de 16,67 (DP = 8.23), variando entre um mínimo de 0 e um máximo de 38 anos de serviço no atual nível de ensino.

\section{Instrumento}

O instrumento utilizado é constituído por dois grupos de questões (cf. anexo A). O primeiro grupo destina-se à avaliação do eustress (Eustress Scale - O'Sullivan, 2011, versão traduzida e adaptada) e é constituído por 15 itens, 10 itens que avaliam o eustress (itens 1, 2 - invertido, 4, 5, 6, 8, 10 - invertido, 12, 13, 15) e 5 questões de despiste (itens $3,7,9,11,14)$. Os itens são avaliados numa escala de tipo Likert de 6 pontos em que: $0=$ Nunca, 1 = Quase Nunca, 2 = Às Vezes, 3 = Frequentemente, $4=$ Muito Frequentemente, 5 = Sempre. O segundo grupo de questões pretende reunir dados pessoais e profissionais dos participantes: sexo, idade, tipo de instituição, nível de ensino a que leciona, tempo de serviço docente e tempo de serviço docente no atual nível de ensino.

\section{Procedimento}

O processo para a adaptação e estudo psicométrico preliminar da Escala de eustress para professores portugueses foi concretizado em diversas etapas que descreveremos a seguir.

5 As percentagens na amostra não correspondem, em proporção, às percentagens existentes na população (32\% sexo masculino e $68 \%$ sexo feminino).

6 As percentagens na amostra não correspondem, em proporção, às percentagens existentes na população $(85,7 \%$ instituições públicas e 14,3\% instituições privadas).

7 As percentagens na amostra não correspondem, em proporção, às percentagens existentes na população $\left(16,3 \% 1^{\circ} \mathrm{CEB}\right.$, $17,3 \% 2^{\circ} \mathrm{CEB}, 45,7 \% 3^{\circ} \mathrm{CEB}$ e Secundário, $20,7 \%$ do Ensino Superior). 
Fase 1: Processo de tradução

Nesta fase procurámos salvaguardar os requisitos científicos do processo de tradução, tendo-se utilizado o método "traduz-retraduz" (Hill \& Hill, 2005) para o efeito. Assim, num primeiro momento, um professor de inglês de origem portuguesa traduziu o questionário original para português; de seguida, um professor inglês, que leciona português a estrangeiros, traduziu a versão portuguesa para inglês; e, num terceiro momento, realizou-se uma comparação da versão original da escala com a versão traduzida para inglês, cujo acordo alcançado se situa por volta dos $80 \%{ }^{8}$, tendo havido apenas necessidade de pequenos ajustamentos sugeridos por ambos os tradutores.

Fase 2: Processo de adaptação

No sentido de adaptar os itens da escala para a população do nosso estudo realizaram-se reflexões faladas com 5 professores ( 1 do ensino superior politécnico, 2 do $3^{\circ}$ ciclo e secundário, 1 do $1^{\circ}$ ciclo e 1 do $2^{\circ}$ ciclo). Das reflexões resultaram contributos para a alteração de alguns termos utilizados na escala, nomeadamente a substituição do termo "académica" pelo termo "profissional" (itens 1, 2, 4, 5, 8, $10,12,13)$ e da expressão "resultados de um teste" pela de "desempenho profissional" (item 15).

A recolha de dados foi realizada entre agosto e setembro de 2011, com recurso à ferramenta Google Docs. O contacto foi efetuado por via eletrónica com os diretores de diversas instituições de ensino, aos quais se solicitou o reencaminhamento do link de resposta à escala, para todos os professores das instituições que dirigiam. Foi ainda realizado um contacto informal, através do facebook, onde se divulgou o referido link e se solicitou a todos os professores portugueses que respondessem e procedessem ao reencaminhamento do mesmo.

\section{Análise dos dados}

Os dados recolhidos através do questionário foram analisados com recurso ao Statistical Package for the Social Sciences - SPSS (versão 20.0) e envolveu duas fases.

Numa primeira fase, efetuaram-se análises prévias de forma determinar a normalidade da distribuição e verificar a existência de outliers suscetíveis de influenciar significativamente os resultados e, com o propósito de averiguar a validade da escala e a sua qualidade psicométrica, foi utilizada a análise fatorial exploratória (método das componentes principais) para definir a estrutura subjacente à escala extraindo um fator, bem como resumir e reduzir os dados (Hair, Anderson, Tatham \& Black, 2005). Para aferir a consistência interna do fator extraído foi também calculado o valor alpha de Cronbach.

8 Calculada a partir do número de diferenças encontradas entre as duas versões das escalas (original e traduzida para inglês) e o acordo final dos tradutores em relação às opções a tomar face às referidas diferenças. 
Na segunda fase realizaram-se análises mais gerais, de carácter descritivo, comparações de médias através de testes t-Student e ANOVA e correlações entre variáveis através do coeficiente de correlação de Pearson.

\section{Resultados}

A análise da distribuição através do teste de Kolmogorov-Smirnov revelou não estarem cumpridos os pressupostos da normalidade $(p<.05)$, o que de acordo com Pallant (2001) é bastante comum em grandes amostras. Pela análise da assimetria e curtose foi possível constatar que estas não são iguais a zero, sendo que os valores da assimetria se situam, para a globalidade dos itens, entre -.524 e .625 e os valores da curtose se situam entre -.410 e 1.755. Contudo, Pallant (2001) relembra que esta ocorrência é comum em investigações na área das ciências sociais. Verificou-se ainda a inexistência de outliers, o que indica que não existem valores extremos nas respostas dos participantes.

\section{Propriedades psicométricas da Escala de Eustress}

Foi testada a dimensionalidade do instrumento ${ }^{9}$ recorrendo-se ao método de análise fatorial exploratória em componentes principais. Para averiguar a viabilidade da utilização desta análise estatística foi utilizado o teste KMO (Kaiser-Meyer-Olkin Measure of Sampling Adequacy) tendo-se obtido um valor de .873, considerado um valor bastante satisfatório, segundo Hair, Anderson, Tatham e Black (2005). $O$ resultado do teste de esfericidade de Bartlett permite também concluir que a matriz de correlações é significativa $\left(\mathrm{c}^{2}{ }_{(45)}=2705.185 ; \mathrm{p}=.000\right)$ pelo que podemos assumir o método da análise fatorial exploratória como viável.

Numa primeira análise forçou-se a extração de um fator dando conta da definição teórica do conceito, contudo, uma vez que alguns dos itens não reuniam as condições necessárias para serem mantidos foram realizadas análises fatoriais sucessivas no sentido de eliminar itens que apresentassem: a) valores de comunalidades inferiores a .45; e b) saturações inferiores a .40 (Stevens, 1986). No final deste processo contínuo de análises, foram retirados os seguintes itens: 1 - Com que frequência lida eficazmente com mudanças stressantes que ocorrem na sua vida profissional? (.187); 2 - Com que frequência falha numa tarefa profissional quando está sobre pressão? (.247); 4 - Com que frequência lida com êxito com dificuldades profissionais irritantes? (.216); 8 - Com que frequência é capaz de controlar com êxito as irritações na sua vida

9 As questões de despiste (filler questions) não visam analisar o constructo de eustress, servem para reduzir a probabilidade de surgirem enviesamentos nas respostas dadas pelos participantes, face ao conteúdo similar dos itens da escala. 
profissional? (.285); e 10 - Com que frequência se sente incapaz de controlar a forma como gere o tempo para a realização de tarefas profissionais? (.134), cujos valores de comunalidade não cumprem o valor critério referido anteriormente.

A eliminação do item 10 é ainda suportada pelos valores de correlações obtidos entre o item e o total da escala, que se apresenta como negativo e não significativo ( $\mathrm{r}=-.077, p=.087$ ). Já no que se refere ao item 2 , apesar de este apresentar uma correlação significativa com o total da escala $(\mathrm{p}<.001)$, o seu valor é baixo e negativo $(\mathrm{r}=-.259)$, reforçando a opção pela sua eliminação (Pestana \& Gageiro, 2008). Importa salientar que estes dois itens são invertidos, podendo ter havido problemas de compreensão por parte dos sujeitos. Na literatura sobre validação de instrumentos, vários autores têm mencionado problemas em relação aos itens invertidos, pois estes exigem mais atenção e compreensão por parte do participante (De Moraes \& Primi, 2002; Giacomoni \& Hutz, 2008).

Os valores das correlações item-total indicam também a existência de correlações baixas e negativas (Pestana \& Gageiro, 2008) para os itens $2(\mathrm{r}=-.378)$ e $10(\mathrm{r}=-.258)$ e correlações baixas mas positivas para os itens $1(\mathrm{r}=-.362), 4(\mathrm{r}=$ -.351) e $8(\mathrm{r}=-.308)$. Assim, qualquer um dos itens acima referenciado, apresenta uma correlação corrigida item-total que apoia a exclusão do respetivo item, por forma a aumentar a consistência interna da escala. Desta forma, do ponto de vista estatístico vale a pena eliminar os cinco itens e do ponto de vista teórico com a sua eliminação não se perde informação relevante, na medida em que o eustress é operacionalizado por O’Sullivan (2011) através de uma estrutura unidimensional, em que todos os itens (com exceção dos itens de despiste) representam o constructo de eustress como um processo e uma consequência desse processo, estando, por esta razão, o conteúdo dos itens eliminados representado nos outros itens da escala.

Retirados os itens 1, 2, 4, 8 e 10 obtivemos resultados satisfatórios que nos permitiram estruturar a escala num fator que na totalidade explica 78,87\% da variância total. O teste $\mathrm{KMO}(.880)$ e o teste de Bartlett $\left(\mathrm{c}_{(10)}^{2}=2142.529 ; \mathrm{p}=.000\right)$ continuaram a ser satisfatórios, assim como os valores das comunalidades. O cálculo do coeficiente alpha de Cronbach para a escala revelou um valor de fidelidade elevado (.93), apontando para uma adequada consistência interna do instrumento (cf. Tabela 1).

Tabela 1.

Estrutura Fatorial Final e Valores Alpha

Componente

1

Com que frequência sente que o stress tem um efeito positivo no seu desempenho profissional? (7) 
Perante uma situação profissional stressante, com que frequência acha que a pressão a/o torna mais produtiva/o? (4)

Com que frequência sente que teve um melhor desempenho numa tarefa profissional, quando sujeito a pressão? (5)

.905

Com que frequência se sente motivado/a pelo seu stress? (2)

.895

Com que frequência sente que o stress contribui positivamente para a sua capacidade de lidar com os problemas profissionais? (1)

Valores próprios

862

Variância explicada (\%)

.839

Alpha de Cronbach

78,87

Nota. O método de extração de fatores utilizado foi a análise de componentes principais

\section{Resultados de carácter descritivo}

Em média, os participantes revelaram que quase nunca e às vezes sentem eustress $(M=1.60, D P=.99)$.

Nas análises comparativas entre grupos, com base no sexo, verificou-se (recorrendo ao teste t-Student para amostras independentes) que não existem diferenças estatisticamente significativas $\left(t_{(170)}=-1.324, p=.187\right)$. Depois de calculado o tamanho do efeito para a população, concluiu-se que a variável sexo tem pouca influência na perceção de eustress $\left(\eta_{p}^{2}=.01\right)$ (Maroco, 2010). Quanto ao tipo de instituição, constatou-se que as diferenças encontradas eram significativas $\left(t_{(60}\right.$ $=-2.682, p<.01)$, sendo a dimensão do efeito média $\left(\eta_{p}^{2}=.11\right)$ (Maroco, 2010). Verificou-se assim, que os professores de instituições privadas $(M=3.05, D P=$ 1.12) experienciam mais eustress do que os professores de instituições públicas $(M=2.57, D P=0.97)$. Através do cálculo do coeficiente de correlação de Pearson verificou-se que a idade e o eustress, na nossa amostra, não estão significativamente associados $(r=-0.19, p=.675)$. No que se refere ao tempo de serviço e ao tempo de serviço no atual nível de ensino verificou-se que não estão significativamente associados ao eustress $(r=-.031 ; \mathrm{n}=496 ; p=.487)(r=-.038 ; \mathrm{n}=496 ; p=.395)$. Quanto às diferenças de médias entre os diversos níveis de ensino a que o professor leciona (utilizado o procedimento One-Way ANOVA, depois de verificado que não era violado o pressuposto de homogeneidade das variâncias) encontraram-se diferenças significativas $\left(F_{(4,491)}=3.34 ; p=.054\right)$. Depois de calculado o tamanho do efeito para a população, concluiu-se que a variável nível de ensino tem pouca influência na perceção de eustress $\left(\eta_{p}^{2}=.02\right)$ (Maroco, 2010). Assim, o teste de Tukey HSD (honestly significant difference) efetuado, para contrastes simples a posteriori, demonstrou diferenças significativas apenas entre os professores do $2^{\circ}$ $\mathrm{CEB}$ e os professores do $3^{\circ} \mathrm{CEB}$ e Secundário $(p=.038)$, apontando para níveis mais elevados de perceção de eustress por parte dos segundos. 


\section{DISCUSSÃO}

Neste estudo procurou-se estudar as características psicométricas da Escala de eustress sob a perspetiva do constructo como um processo e uma consequência/ produto (O’Sullivan, 2011; Nelson \& Simmons, 2003, 2005; Simmons, 2000; Simmons \& Nelson, 2001), tal como é definido pela autora da escala e operacionalizado neste instrumento de medida (O’Sullivan, 2011). Com base nos resultados obtidos podemos assumir que, a Escala de eustress resultante deste estudo permite, para esta amostra, ser considerada válida o que possibilita a sua utilização para futuras investigações sobre o tema com professores portugueses e que o constructo eustress deve ser entendido como um processo e uma consequência/produto da resposta ao stress. $\mathrm{O}$ instrumento revelou propriedades psicométricas adequadas no que concerne à sua consistência interna $(\alpha=.93)$. Estes valores são mais elevados que o encontrado pelo autor da escala $(\alpha=.806)$ (O'Sullivan, 2011) e reforçam os resultados obtidos num estudo realizado por Fonseca e Jordão (2011), onde se utilizou a Escala de eustress com 5 itens, com uma amostra mais pequena e independente da utilizada neste estudo $(\alpha=.95)$.

A análise fatorial exploratória permitiu constatar que o fator explica uma percentagem considerável da variância $(78,87 \%)$ e que a estrutura da escala deve apresentar apenas 7 itens (5 itens que saturam o fator e 2 questões de despiste ${ }^{10}$ ). Este resultado vem reforçar o já obtido por Fonseca e Jordão (2011) com uma amostra mais pequena e independente.

Procurou-se, também, neste estudo descrever o nível de eustress percecionado pelos professores inquiridos, perceber se existem diferenças em função das variáveis pessoais e profissionais consideradas e conhecer a relação entre as variáveis em estudo e, neste sentido, os resultados obtidos apontam para o facto de que os professores portugueses não experienciam eustress com muita frequência (em média, os participantes responderam que quase nunca e às vezes experienciam eustress), contrariamente aos resultados obtidos em outros grupos profissionais (p. ex., enfermeiros) que apontam para níveis mais elevados de eustress percecionados (Nelson \& Simmons, 2005; Simmons, 2000; Simmons \& Nelson, 2001; Simmons, Nelson, \& Quick, 2003; Simmons, Nelson, \& Neal, 2001).

Os participantes de instituições privadas experienciam mais eustress, assim como, os professores do $3^{\circ} \mathrm{CEB}$ e Secundário em relação aos professores do $2^{\circ}$ CEB. Estas diferenças poderão advir das características específicas das políticas de contratação das instituições de ensino públicas e privadas, na medida em que

10 Decidiu-se reduzir o número de questões de despiste de 5 para 2, em proporção à redução no número de itens, decorrente da análise fatorial realizada. A seleção das duas questões de despiste foi aleatória. 
os professores que lecionam em instituições públicas estão sujeitos a políticas de contratação mais rígidas, subjacentes a concursos públicos nacionais e a sistemas de avaliação de desempenho anuais (Araújo \& Jordão, 2011). As diferenças mencionadas podem ainda advir de aspetos associados às características intrínsecas ao trabalho e ao(s) papel/papéis desempenhado(s) (Cartwright \& Cooper, 1997), decorrentes do "poder" que o Ministério da Educação detém em relação às escolas públicas, que se repercute em políticas que determinam, entre outros aspetos, o aumento do número de alunos por turma e o aumento do trabalho burocrático realizado pelos professores (Kelly, 2014) .

\section{Limitações}

Os resultados obtidos e as conclusões retiradas dos mesmos devem ser consignados à amostra em estudo, na medida em que esta não é representativa da população.

Uma outra limitação do estudo apresentado prende-se com o facto de os dados terem sido recolhidos num período em que não havia aulas (agosto e setembro), podendo este facto ter influenciado as respostas dadas, na medida em que a maioria dos estudos existentes na literatura apontam como fontes indutoras de stress (distress e eustress) aspetos diretamente relacionados com o desempenho de funções letivas, tais como, o contacto com os alunos, os momentos de avaliação, a indisciplina e o confronto com desmotivação dos alunos (Gomes et al., 2006; Kyriacou, 1987; Kyriacou, 1998; Kyriacou, 2001; Maia, 2004; Mota-Cardoso et al., 2002; Travers \& Cooper, 1996).

\section{Pistas futuras de investigação}

Futuramente, importa aprofundar os estudos psicométricos da escala realizando uma análise da estabilidade da mesma (teste-reteste).

Além disso, seria também importante controlar o tipo de contrato do professor, aspeto que pode influenciar a perceção de eustress, na medida em que diversos autores destacam a precaridade, inerente ao tipo de contrato, como uma importante fonte indutora de stress dos professores (Maia, 2004; Teodoro, 1994), e por contingências inerentes aos objetivos primordiais do nosso estudo, não foi controlado.

Importa ainda, no futuro, para uma compreensão integradora do fenómeno do stress ocupacional nos professores portugueses, e sob a orientação do modelo holístico de stress (Nelson \& Simmons, 2003), avaliar concomitantemente o eustress e o distress (Simmons, 2000), na medida em que, como demonstram diversos estudos, o eustress é mais do que a mera ausência de distress, que as respostas aos stressores podem ser quer positivas quer negativas e que para conhecer verdadeiramente 
o fenómeno stress ocupacional, devemos atender às suas duas vertentes (Campbell-Quick et al., 2003; Le Fevre et al., 2003; Nelson \& Simmons, 2003; Nelson \& Simmons, 2005; Simmons, 2000).

Deste modo, a adaptação, validação e exploração das propriedades psicométricas da Escala de eustress, permite que no futuro sejam desenvolvidos trabalhos que contemplem uma compreensão alargada e integradora do stress nos professores portugueses, considerando este instrumento e outros que permitam avaliar o distress nesta população. No que se refere aos resultados obtidos, nos professores em relação ao distress, os estudos indicam que os professores experienciam níveis moderados a elevados (Gomes \& Cruz, 2004; Gomes et al., 2006; Kyriacou, 1987; Kyriacou, 1998; Kyriacou, 2001; Maia, 2004; Melo, Gomes, \& Cruz, 1997; Mota-Cardoso et al., 2002; Travers \& Cooper, 1996; Venâncio et al., 2000). Estes resultados, e os por nós obtidos neste estudo, parecem indicar que os professores experienciam mais distress do que eustress, contudo, importa futuramente aprofundar esta questão, conjugando o estudo destes dois constructos numa mesma amostra.

Podemos concluir, assim, que este trabalho constitui-se como um contributo para o estudo do stress em geral (considerando uma perspetiva integradora e holística do mesmo) e, em particular, do eustress, ao providenciar um instrumento de avaliação deste constructo para professores portugueses.

\section{REFERÊNCIAS}

Araújo, P., \& Jordão, F. (2011). “Os inempregáveis”: estudo de caso sobre os impactos psicossociais do não-emprego em licenciados portugueses. Análise Psicológica, 2(XXIX), 289-314.

Benson, H., \& Allen, R. (1980). How much stress is too much? Harvard Business Review, 58, 86-92.

Bishayee, S. (2012). Job-stress of faculty members in private professional colleges located in NCR, DELHI. International Journal of Research in Commerce, Economics \& Management, 2(6), 37-39.

Campbell-Quick, J., Cooper, C., Nelson, D., Quick, J., \& Gavin, J. (2003). Stress, health and wellbeing at work. In J. Greenberg (Coord.), Organizational Behaviour: The State of Science (pp. 53-89). Mahwah: Lawrence Eribaum Associates.

Cartwright, S., \& Cooper, C. (1997). Managing Workplace Stress. USA: Sage Publications.

Certo, S. (2003). Supervision: Concepts and Skill Building (4a ed.). New York: McGraw Hill.

Cohen, S., Kamarck, T., \& Mermelstein, R. (1983). A global measure of perceived stress. Journal of Health and Social Behavior, 24, 385-396.

Cooper, C. (1998). Theories of Organizational Stress. New York: Oxford University Press Inc.

Cooper, C., Dewe, P., \& Driscoll, M. (2001). Organizational stress - a review and critique of theory, research and applications. USA: Sage Publications.

Cummings, T., \& Cooper, C. (1998). A cybernetic theory of organizational stress. In C. Cooper (Ed.), Theories of Organizational Stress (pp. 101-21). New York: Oxford University Press. 
De Moraes, I., \& Primi, R. (2002). Escala de avaliação de tipos psicológicos: validade e precisão. Psico-USF, 7(1), 25-34.

Edwards, J., Caplan, R., \& Van Harrison, R. (1998). Person-environment fit theory: conceptual foundations, empirical evidence, and directions for future research. In C. Cooper (Ed.), Theories of Organizational Stress (pp. 28-67). New York: Oxford University Press.

Edwards, J., \& Cooper, C. (1988). The impacts of positive psychological states on physical health: A review and theoretical framework. Social Science \& Medicine, 27, 1447-1459.

Folkman, S. (2008). The case for positive emotions in the stress process. Anxiety, Stress \& Coping, 21(1), 3-14.

Fonseca, S., \& Jordão, J. (2011, novembro). Validação da Escala de Eustress para Professores Portugueses. Comunicação apresentada no II Congresso Internacional Interfaces da Psicologia - Qualidade de Vida... Vidas de Qualidade, Évora.

Giacomoni, C., \& Hutz, C. (2008). Escala multidimensional de satisfação de vida para crianças: estudos de construção e validação. Estudos em Psicologia (Campinas), 25(1), 25-35.

Gillespie, N., Walsh, M., Winefield, A., Dua, J., \& Stough, C. (2001). Occupational stress in universities: staff perceptions of the causes, consequences and moderators of stress. Work \& Stress, 15(1), 53-72.

Gomes, A., \& Cruz, J. (2004). A experiência de stress e burnout em psicólogos portugueses: um estudo sobre as diferenças de género. Psicologia: Teoria, Investigação e Prática, 2, 193-212.

Gomes, A. R., Silva, M. J., Mourisco, S., Silva, S., Mota, A., \& Montenegro, N. (2006). Problemas e desafios no exercício da actividade docente: Um estudo sobre o stresse, "burnout”, saúde física e satisfação profissional em professores do $3^{\circ}$ ciclo e ensino secundário. Revista Portuguesa de Educação, 19(1), 67-93.

Hair, J., Anderson, R., Tatham, R., \& Black, W. (2005). Análise multivariada de dados. São Paulo: Bookman.

Hill, M., \& Hill, A. (2005). Investigação por Questionário. Lisboa: Edições Sílabo.

Karasek, R. (1979). Job demands, job decision latitude, and mental strain: Implications for job redesign. Consultado em http://www.jstor.org/discover/10.2307/2392498?uid=3738880\&uid=2134\&uid=377731553 \&uid=2\&uid=70\&uid=3\&uid=377731543\&uid=60\&purchase-type=article\&accessType=none\&sid=21$101591686713 \&$ showMyJstorPss $=$ false $\&$ seq $=2 \&$ showAccess $=$ false

Kelly, M. (2014). Teaching at private vs. public schools. Looking at the differences between private and public schools. Consultado em http://712educators.about.com/od/jobopenings/a/private-public.htm

Kyriacou, C. (1987). Teacher stress and burnout: An international review. Educational Research, 29, 146-152.

Kyriacou, C. (1998). Teacher stress: Past and Present. In J. Dunham \& V. Varma (Eds.), Stress in Teachers. Past, present and future (pp. 1-13). London: Whurr Publishers Ltd.

Kyriacou, C. (2001). Teacher stress: Directions for future research. Educational Research, 53(1), 27-35.

Kyriacou, C., \& Sutcliffe, J. (1979). A note on teacher stress and locus of control. Journal of Occupational Psychology, 52, 227-228.

Lazarus, R., \& Folkman, S. (1984). Stress, appraisal and coping. New York: Springer.

Le Fevre, M., Matheny, J., \& Kolt, G. (2003). Eustress, distress, and interpretation in occupational stress. Journal of Managerial Psychology, 18, 726-744.

Little, L., Simmons, B., \& Nelson, D. (2007). Health among leaders: positive and negative affect, engagement and burnout, forgiveness and revenge. Journal of Management Studies. Special Issue Managerial Dimensions of Organizational Health: The Healthy Leader at Work, 44, 243-260. 
Maia, M. C. (2004). O stresse e a organização escolar (Dissertação de mestrado). Universidade de Aveiro, Aveiro.

Maroco, J. (2010). Análise estatística. Com utilização do SPSS (3ª Ed.). Lisboa: Edições Sílabo, Lda.

McGowan, J., Gardner, G., \& Fletcher, R. (2006). Positive and negative affective outcomes of occupational stress. New Zealand Journal of Psychology, 35, 92-98.

Melo, B., Gomes, A., \& Cruz, J. (1997). Stress ocupacional em profissionais da saúde e do ensino. Psicologia: Teoria, Investigação e Prática, 2, 53-71.

Mota-Cardoso, R., Araújo, A., Ramos, R., Gonçalves, G., \& Ramos, M. (2002). O stress nos professores portugueses. Estudo IPSSO 2000. Porto: Porto Editora.

Nelson, D., \& Simmons, B. (2003). Health psychology and work stress: A more positive approach. In J. Quick \& L. Tetrick (Eds.), Handbook of Occupational Health Psychology (97-119). Washington, D. C.: American Psychological Association.

Nelson, D., \& Simmons, B. (2005). Eustress and attitudes at work: a positive approach. In A-S. Antoniou \& C. Cooper (Eds.), Research Companion to Organizational Health Psychology (pp. 102-110). Massachusetts: Edward Elgar Publishing Limited.

O'Sullivan, G. (2011). The Relationship Between Hope, Eustress, Self-Efficacy, and Life Satisfaction Among Undergraduates. Social Indicators Research, 101(1), 155-172.

Pallant, J. (2001). SPSS Survival Manual. Buckingham: Open University Press.

Pestana, M., \& Gageiro, J. (2008). Análise de dados para ciências sociais (5ª ed. rev.). Lisboa: Edições Sílabo.

Quick, J. C., Quick, J. D., Nelson, D., \& Hured, J. (1997). Preventative stress management in organizations. Washington, DC: APA.

Seligman, M., \& Csikszentmihalyi, M. (2000). Positive psychology. American Psychologist, 55(1), 5-14.

Selye, H. (1974). Stress without distress. Philadelphia, PA: Lippincott.

Selye, H. (1976). Stress in health and disease. Boston: Butterworths.

Selye, H. (1987). Stress without Distress. London: Transworld.

Simmons, B. (2000). Eustress at work: Accentuating the positive (Unpublished doctoral dissertation). Faculty of the Graduate College, Oklahoma State.

Simmons, B., \& Nelson, D. (2001). Eustress at work: The relationship between hope and health in hospital nurses. Health Care Management Review, 26(4), 7-18.

Simmons, B., \& Nelson, D. (2007). Eustress at work: Extending the Holistic Stress Model. In D. Nelson \& C. Cooper (Eds.), Positive Organizational Behavior (pp. 40-53). London: Sage Publications.

Simmons, B., Nelson, D., \& Quick, J. (2003). Health for the Hopeful: A Study of Attachment Behavior in Home Health Care Nurses. International Journal of Stress Management, 10(4), 361-375.

Simmons, B., Nelson, D., \& Neal, L. (2001). A Comparison of the Positive and Negative Work Attitudes of Home Health Care and Hospital Nurses. Health Care Management Review, 26(4), 63-74.

Stevens, J. (1986). Applied multivariate statistics for the social sciences. New Jersey: Lawrence Erlbaum. Teodoro, A. (1994). A Carreira Docente. Lisboa: Texto Editora.

Travers, C., \& Cooper, C. (1996). Teachers under pressure. Stress in the teaching profession. London: Routledge.

Venâncio, C., Carmo, R., Mendes, S., Liberato, R., \& César, M. (2000). Stress: Professores à beira de um ataque de nervos!... In E. Fernandes \& J. F. Matos, Actas do ProfMat 2000 (pp. 205-214). Universidade da Madeira: Associação de Professores de Matemática. 
ANEXO A

\section{QUESTIONÁRIO SOBRE STRESS OCUPACIONAL}

Este questionário insere-se no âmbito de um estudo que está a ser realizado na Faculdade de Psicologia e de Ciências da Educação da Universidade do Porto, sendo que agradecemos a sua disponibilidade para o preenchimento do mesmo.

O estudo destina-se exclusivamente a fins de investigação, pelo que está garantida uma absoluta confidencialidade e anonimato no tratamento dos dados.

Não existem respostas corretas ou erradas. Verifique, no final, se respondeu a TODAS as questões.

Desde já, o nosso muito obrigado pela sua colaboração!

Por favor, assinale com uma cruz (x) a opção que melhor corresponde ao seu caso pessoal. Para cada pergunta escolha uma das opções disponibilizadas (Nunca; Quase nunca; Às vezes; Frequentemente; Muito frequentemente; Sempre):

\begin{tabular}{|c|c|c|c|c|c|c|}
\hline & $\underset{\Xi}{\tilde{Z}}$ & 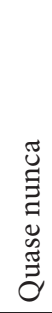 & 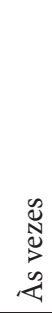 & 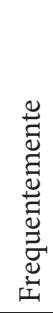 & 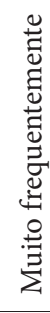 & 苂 \\
\hline $\begin{array}{l}\text { 1. Com que frequência lida eficazmente com } \\
\text { mudanças stressantes que ocorrem na sua vida } \\
\text { profissional? }\end{array}$ & & & & & & \\
\hline $\begin{array}{l}\text { 2. Com que frequência falha numa tarefa profis- } \\
\text { sional quando está sobre pressão? }\end{array}$ & & & & & & \\
\hline 3. Lê livros por prazer? & & & & & & \\
\hline $\begin{array}{l}\text { 4. Com que frequência lida com êxito com dificul- } \\
\text { dades profissionais irritantes? }\end{array}$ & & & & & & \\
\hline $\begin{array}{l}\text { 5. Com que frequência sente que o stress contribui } \\
\text { positivamente para a sua capacidade de lidar com os } \\
\text { problemas profissionais? }\end{array}$ & & & & & & \\
\hline $\begin{array}{l}\text { 6. Com que frequência se sente motivado/a pelo seu } \\
\text { stress? }\end{array}$ & & & & & & \\
\hline 7. Sai com os seus amigos durante a semana? & & & & & & \\
\hline
\end{tabular}




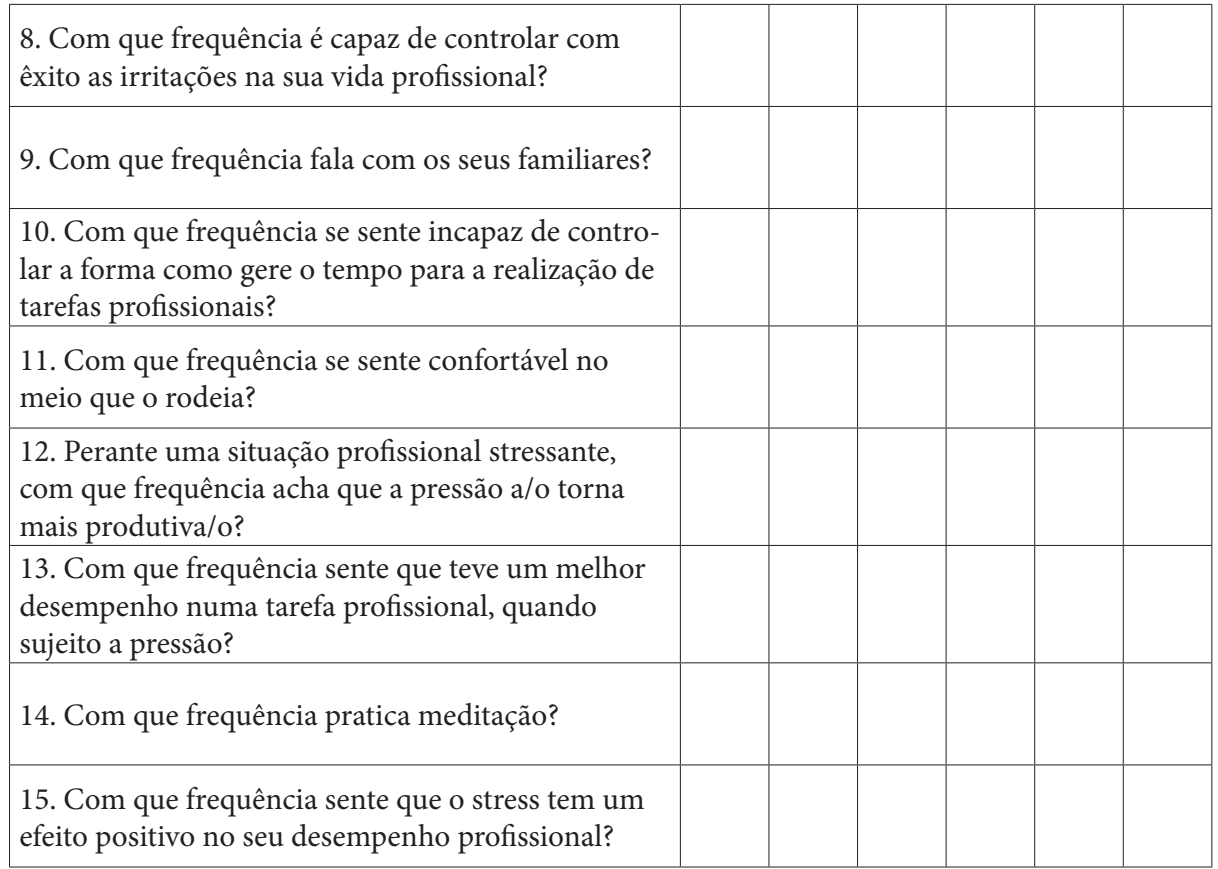

\section{Dados Pessoais e Profissionais}

Sexo: $\quad \mathrm{F} \quad \mathrm{M}$

Idade:

\section{Nível de ensino em que leciona:}

$1{ }^{\circ} \mathrm{CEB}^{*}$

\begin{tabular}{|l|l|l|}
\hline $\begin{array}{l}2^{\circ} \mathrm{CEB} \\
3^{\circ} \mathrm{CEB} \text { e Secundário }\end{array}$ & \begin{tabular}{l} 
Grupo em que leciona: \\
\cline { 2 - 3 }
\end{tabular} & $\begin{array}{l}\text { Público } \\
\text { Privado } \\
\text { Fundação }\end{array}$ \\
\hline Ensino Superior & $\begin{array}{l}\text { Universitário } \\
\text { Politécnico }\end{array}$ & \\
\hline
\end{tabular}

\section{Há quantos anos leciona?}

Há quantos anos leciona no atual nível de ensino?

${ }^{\star} \mathrm{CEB}=$ Ciclo do Ensino Básico

Por favor, verifique se respondeu a todas as questões.

A falha de uma resposta obriga à anulação de todo o questionário. 